\title{
Unifying and Testing Models of Sexual Selection
}

\section{Hanna Kokko, ${ }^{1}$ Michael D. Jennions, ${ }^{2}$ and Robert Brooks ${ }^{3}$}

${ }^{1}$ Department of Biological and Environmental Science, University of Helsinki, FIN-00014 Helsinki, Finland; email: hanna.kokko@helsinki.fi

${ }^{2}$ School of Botany and Zoology, Australian National University, Canberra ACT 0200, Australia; email: michael.jennions@anu.edu.au

${ }^{3}$ School of Biological, Earth, and Environmental Sciences, University of New South Wales, Sydney NSW 2052, Australia; email: rob.brooks@unsw.edu.au

Annu. Rev. Ecol. Evol. Syst. 2006. 37:43-66

First published online as a Review in Advance on July 21, 2006

The Annual Review of Ecology, Evolution, and Systematics is online at

http://ecolsys.annualreviews.org

This article's doi:

10.1146/annurev.ecolsys.37.091305.110259

Copyright (C) 2006 by Annual Reviews.

All rights reserved

1543-592X/06/1201-0043\$20.00

\section{Key Words}

anisogamy, female choice, mate choice, sex roles, sexual conflict

\section{Abstract}

Sexual reproduction is associated with the evolution of anisogamy and sperm-producing males and egg-laying females. The ensuing competition for mates has led to sexual selection and coevolution of the sexes. Mathematical models are extensively used to test the plausibility of different complicated scenarios for the evolution of sexual traits. Unfortunately, the diversity of models is now itself equally bewildering. Here we clarify some of the current debate by reviewing evolutionary explanations for the relationship between anisogamy, potential reproductive rates, parental care, sex roles, and mate choice. We review the benefits females might gain by mating with certain males rather than others. We also consider other forms of selection that can make females mate nonrandomly. One way empiricists can contribute to resolving theoretical disputes is to quantify the cost of expressing mating biases in the appropriate life-history currency. 
Sexual selection: selection generated by differential access to opposite-sex gametes (or mates)

Anisogamy: difference in the size of the gametes that forms the basis of the definition of males and females

\section{INTRODUCTION}

Sexual selection occurs when individuals compete for access to gametes of the opposite sex (Andersson 1994). The kinds of traits it generates are so flamboyant and even devious that sexual selection, despite a component of natural selection, is often elevated to the status of the so-called other force shaping the evolution of organisms.

Sexual selection is the result of interactions between individuals of the same sex, opposite sexes, or both. Males usually produce enough sperm over their lifetime to inseminate many females. Their reproductive success depends primarily on how many females they mate with, the fecundity of these females, and the proportion of each female's eggs they fertilize. Males are therefore under strong sexual selection to improve all three aspects of reproductive success. In addition to the number of available gametes, the payoff of mating can also vary depending on qualitative differences among gametes. Mate choice is additionally under natural selection: It can increase access to material resources required for reproduction (e.g., nesting sites) or other aspects of fitness (although this too can be considered sexual selection, e.g., if the mate phenotype determines access to these resources). Given this complexity, verbal arguments about how sexual selection influences both sexes can be convoluted and are certainly insufficient.

Mathematical modeling has played a major role in the development of the field. It clarifies verbal arguments, exposes hidden assumptions, and tests whether the stated outcomes actually emerge from the starting premises. Unfortunately, this has led to a proliferation of superficially identical models, making it difficult to see the forest for the trees. Claims and counterclaims about whether specific processes can occur (e.g., compare Kirkpatrick 1986 and Grafen 1990a) have caused some biologists to argue that theoretical models make inconsistent and often transitory claims about what is possible in the real world (Eberhard 2005). In reality, if models reach different conclusions, they must be making different assumptions. Differences should therefore be considered illuminating. It is the empiricist's task to test assumptions directly or to argue whether they are biologically plausible. The view that modelers are divorced from reality is strengthened when theoretical models are used to make blanket statements. For example, Maynard Smith (1977) claimed that paternity cannot have an effect on paternal care because similar reductions of paternity would occur in future broods. Subsequent empirical studies tend to find, however, that care increases with a greater share of paternity (Sheldon 2002), and more recent models have shown this to be the theoretical expectation when paternity varies between broods (Houston \& McNamara 2002).

The sexual-selection literature is enormous, covering a gamut of specialized topics (e.g., fighting assessment, signaling, sperm competition, intragenomic conflict, sex ratio skew, and differential allocation). Instead of attempting a comprehensive review of every available model, we begin with the foundations of sexual selection (anisogamy and parental roles) and then focus on the single most-debated effect of sexual selection: the evolution of mate choice for elaborate traits. We hope to show that the field could benefit from conceptual unification of several key areas that have developed in partial isolation. 


\section{SEXUAL SELECTION AND SEX ROLES: IS YOUR TEXTBOOK RIGHT?}

Sex and the fusion of gametes are prerequisites for sexual selection. The evolution of sexual reproduction per se is beyond the scope of our review, but it is interesting to note that sexual selection might help maintain sexual reproduction (Agrawal 2001, Siller 2001). Once there is sexual reproduction with two different mating types, distinct reproductive strategies can evolve. Typically one type provides offspring with fewer material benefits. The simplest case of unequal investment is a difference in gamete size (anisogamy). The sex producing smaller gametes is, by definition, the male. This is why seahorses that become pregnant after insemination are still referred to as males: They produced the smaller gametes (Berglund \& Rosenqvist 2003).

Although anisogamy is sometimes argued to have evolved to ensure uniparental inheritance of cytoplasmic elements (Randerson \& Hurst 2001), its evolution is more-widely attributed to disruptive selection. Two main assumptions are required: (a) Smaller gametes can be produced in larger numbers, and (b) larger zygotes with more resources survive better (Parker et al. 1972). Intermediate-sized gametes fall in between these two advantages, and, assuming suitable survival functions, disruptive selection arises when it is best to produce either small searcher or large resourceprovider gametes (Bulmer \& Parker 2002). In many species, this leads to individuals that are exclusively male or female and specialize in producing a single gamete type, although limited mate availability can also select for simultaneous hermaphrodites (Puurtinen \& Kaitala 2002).

Even if the total biomass of gametes produced by each sex is equal, anisogamy implies that abundant sperm compete for far less numerous eggs. This competition creates a race among males to ensure their sperm locate eggs and fertilize them before those of rivals: Sexual selection is born. In external fertilizers, it selects for mechanisms to optimize the timing and quantity of sperm released (Levitan 2004) and for improved sperm efficiency in locating and entering eggs. If males are mobile, it selects for improvements in their ability to track down females; if females are mobile, it selects for males that increase the ease with which females locate them and the likelihood that they mate with them. When mating encounters allow sperm from several males to have the potential to fertilize eggs-multiple mating in internal fertilizers (Jennions \& Petrie 2000) or simultaneous sperm release by adjacent males in external fertilizers (Byrne et al. 2003) —selection favors traits of males or their sperm that increase the prospects of fertilizing more eggs than their rivals (Simmons 2001). All these traits evolve through sexual selection initiated by anisogamy.

The relative size difference between sperm and eggs is large, but, in absolute terms, the difference in gamete-production costs can be trivial compared with those of other reproductive activities, such as lactation by female mammals or intense calling by male frogs. Still, there are good reasons to believe that anisogamy fundamentally influences sexual dimorphism in these traits. The standard textbook explanation for why females care for offspring and males seek out additional mates runs as follows: Anisogamy allows males to invest initially less per reproductive event. They therefore have a higher potential reproductive rate (PRR); hence they gain more than
Potential reproductive rate (PRR): the highest possible reproduction rate for an individual given the constraints of speed of gamete production and parental care and assuming unlimited access to opposite-sex mates 
Operational sex ratio (OSR): the ratio of male versus female individuals that are available for mating at any given time females from deserting their current offspring if there is a trade-off between caring for current young and finding new mates. This amplifies the initial asymmetry: Males that provide less parental investment are selected to provide even less, and females compensate by increasing their investment (Clutton-Brock \& Parker 1992, Emlen \& Oring 1977, Maynard Smith 1977, Trivers 1972, Williams 1966). The result is a male-biased operational sex ratio (OSR), which forces males to compete even more intensely for mates and allows females to divert investment from sexual competition into providing more care for their offspring. It also permits females to be choosy, as they constantly encounter eager males. Subsequent models have refined this general theme, for example, by pointing out that choosiness is not a perfect mirror image of competition for mates, as choosiness depends on variation in the quality of potential mates (Johnstone et al. 1996, Owens \& Thompson 1994).

Queller (1997), in a paper largely ignored at the time, questioned the logic of the standard explanation for asymmetries in parental care. His insight was that it is inconsistent to claim that the higher PRR of males allows them to gain more than females from searching for additional mates. The PRR is only a potential rate. In a population with a male-biased OSR, few males ever achieve anything near their potential. More generally, each offspring in diploid species has one father and one mother (Fisher 1930). This so-called Fisher condition (sensu Houston \& McNamara 2005) guarantees that the number of offspring produced by males cannot exceed that produced by females. Anisogamy may lead to an asymmetry in PRR, but this cannot explain why females provide more care than males (see also Kokko \& Jennions 2003).

Queller (1997) pointed out two ways to escape this dilemma. First, multiple mating and sperm competition create uncertainty about paternity for males, diminishing the expected fitness gain of caring for young. In females, uncertainty about maternity only occurs in rare circumstances such as egg dumping (Tallamy 2005). Second, a subset of males may be consistently more likely to mate than others if their traits are favored by sexual selection. Under a male-biased OSR with some males consistently more successful than others, the future reproductive rate of a breeding male is higher than that of the average male. Because average male reproductive rates equal average female reproductive rates, a successful male reproduces faster than the average breeding female. If success is nonrandom, the costs of caring owing to lost mating opportunities are therefore higher for males than for females. Interestingly, all these points have been made by those using PRR to predict the direction of sexual selection (e.g., Parker \& Simmons 1996). However, before Queller (1997), they were never fully integrated with the evolution of parental care. (A final possibility is that the adult sex ratio is female-biased so that males must have a higher mating rate. To be a distinct explanation, however, this bias cannot be a result of sexual selection.)

In summary, anisogamy does not lead to different male-female parental roles because differential investment per gamete creates a sexual asymmetry in potential mating rates. The causal route is instead that anisogamy generates reproductive competition among males. The resultant sexual selection, including that owing to sperm competition, leads to uncertainty of paternity as well as predictable differences in reproductive rates between females and a subset of reproductively successful males. 
These two factors make it more likely that males will pursue additional mating opportunities rather than invest in their current offspring.

Interestingly, Queller's insights coincided with growing recognition that models should be self-consistent. It is trivially true that models must be internally consistent (the books must balance; for example, the Fisher condition must hold so that every offspring has the correct number of parents), but there is also a growing trend to ensure that models of specific phenomena are also consistent with the wider system in which they are embedded (Houston \& McNamara 2005). The way to ensure this is to avoid fixing parameters and instead let them emerge from the model. For example, recent models of caring do not fix the value of carer-offspring relatedness or caring payoffs. Instead these values change as a result of the population-level pattern of desertion, mortality, and migration (e.g., Härdling et al. 2003, Houston \& McNamara 2002, Houston et al. 2005, Webb et al. 1999). Problems with consistency are nevertheless often overlooked, as illustrated by some recent models purporting to fix the problem (Wade \& Shuster 2002) that in fact do not (Houston \& McNamara 2005).

\section{WHO CHOOSES AND WHO COMPETES?}

Isogamous species tend to be unicellular; only multicellular species are anisogamous (Randerson \& Hurst 2001). This makes testing whether the evolution of anisogamy promotes divergent sex roles empirically intractable: It is difficult to envisage how sex roles could develop or be measured in unicellular species independently of gamete size. Fortunately, anisogamy does not always produce classical sex roles. Competing for mates and being choosy are not mutually exclusive alternatives, and several species of birds, fish, frogs, and insects show either permanent or context-dependent sex-role reversal. Studies of these species provide an empirical testing ground to clarify what factors other than anisogamy drive sexual differences in choosiness, competitiveness, and mate-searching behavior (e.g., Barlow 2005, Simmons \& Kvarnemo 2006).

Prior to Queller (1997), models simply assumed that the effect of relative parental investment on the PRR and OSR would predict which sex competed for mates (Clutton-Brock \& Parker 1992, Parker \& Simmons 1996) and, by extension, that the other sex was choosy. The insight that factors such as variance in mate quality also influence these predictions (Johnstone et al. 1996, Owens \& Thompson 1994), as well as the requirement of self-consistency, has prompted new research into sex-role reversal and choosiness by both sexes (Kokko \& Johnstone 2002, Kokko \& Monaghan 2001). These models refrain from assuming that a higher male PRR and male-biased OSR equate to greater competition among males. Instead, these models employ a life-history approach to calculate the sex-specific fitness consequences of varying the investment into competition and/or choosiness.

The predictions are similar to those of earlier models, but there are subtle differences. The most important is that the cost of breeding can be seen as a morefundamental factor than either the OSR or PRR in determining which sex competes more intensely for mates (Kokko \& Monaghan 2001). This is best illustrated by the 
finding that the OSR at which the intensity of competition is greater in males than females need not be unity. Despite a female-biased OSR, males may still compete more intensely for mates if breeding is costlier for females (Kokko \& Monaghan 2001, equation 4). Even so, the familiar relationships between the OSR, PRR, and competition for mates are often still present. The cost of breeding increases if there is a greater rate of adult mortality while breeding and/or if it takes longer to breed, and the OSR is influenced by the effect of breeding on adult mortality and the PRR by the time taken to re-enter the pool of potential mates. Therefore, a relatively higher breeding cost for females, unless balanced by greater nonbreeding male mortality, is associated with a male-biased OSR and higher male PRR. The statement that the costs of breeding are pivotal in determining which sex competes does not preclude other factors as better predictors in specific cases. If, for example, the adult sex ratio is female-biased for reasons unrelated to breeding costs, then females might still compete for males even if breeding was costlier for them.

The value of these more-recent models depends on perspective. On one hand, they make fewer implicit assumptions, thus providing a more-integrated picture of how sex roles, choosiness, and parental investment coevolve. Investigating additional components also increases predictive power, such as predicting the switch point in species that show conditional sex-role reversal (e.g., Forsgren et al. 2004, Simmons \& Kvarnemo 2006). On the the other hand, if everything interacts with everything, it is difficult to make general predictions that are amenable to empirical tests because so many parameters have to be measured. But that is life.

There is always a tension between general heuristic models and specific models of particular systems. A clear trend exists in sexual-selection modeling toward more-inclusive models that consider future mating prospects in a self-consistent way and include feedback between choice (by both sexes), parental care, and mating effort (Houston et al. 2005, McNamara et al. 1999). Instead of fixing parameters, these models allow interactions between individuals to generate parameters (sex ratio, availability of mates) that can change over time (e.g., Alonzo \& Warner 2000). Often these models are more complex than their predecessors, but there is a good reason. Houston et al. (2005) have argued that earlier models sometimes oversimplified to the point of being misleading. Empiricists need to recognize that modeling, similar to empirical data collection, progresses. Conclusions are made on the basis of the information and techniques available at the time. The influential models of Maynard Smith (1977) alerted us all to conflicts inherent in parental care, even if they do not satisfy current standards of consistency (Houston \& McNamara 2005).

\section{UNIFICATION OF MODELS OF THE EVOLUTION OF MATE CHOICE}

Lande (1981) offered a breakthrough genetic model of the potential for runaway selection of male ornaments. In so doing he fleshed out an idea briefly sketched by Fisher (1930) a half-century earlier. Another quarter-century of subsequent modeling has led to a bewildering variety of evolutionary scenarios of how and when mate choice 
will evolve. There are still unresolved disputes as to the number of distinct processes involved in mate-choice evolution and their relative importance.

Mate choice is the outcome of the inherent propensity of an individual to mate more readily with certain phenotypes of the opposite sex (i.e., mating preference or bias) and the extent to which an individual engages in mate sampling before deciding to mate (i.e., choosiness). Here we pool these two aspects of choice, simply noting that preferences might be cost-free, whereas choosiness is less likely to be so.

The evolutionary trajectory of mate choice, similar to that of any other trait, depends on genetic variation in and the strength of direct selection on choice, and indirect selection that relies on genetically correlated traits. The strength of selection is proportional to the covariance between fitness and a trait. Fuller et al. (2005) recently provided a simplified multivariate, quantitative genetic framework to discuss the various evolutionary forces acting on mate choice, and we follow their approach here. In a few cases we have made relationships more explicit (e.g., in the absence of indirect selection and mutation, two forces of direct selection on a trait must be opposite and equal at equilibrium). Pomiankowski \& Iwasa (2001) provide a morerigorous mathematical review of these models.

The change in the mean values of a set of traits after a generation of selection $(\Delta \overline{\mathbf{z}})$ can be expressed as

$$
\Delta \overline{\mathbf{z}}=\mathbf{G} \beta+\mathbf{u} .
$$

Here $\mathbf{G}$ is the matrix of genetic variances in and covariances among a set of traits (reviewed in Steppan et al. 2002). The vector of selection gradients, $\beta$, describes the intensity of direct selection on each trait, whereas $\mathbf{u}$ describes the extent to which each trait's mean values change owing to mutation (Lande 1979, Lande \& Arnold 1983). Theory suggests, and empirical evidence shows, that mutations contribute more to the genetic variance of some traits than others. This is partly a result of the number of genes influencing a trait and the timing of their expression (Houle et al. 1996, Rifkin et al. 2005). This should alert us to the fact that $\mathbf{u}$ and $\mathbf{G}$ are not independent. Phenotypic change in traits (e.g., age when expressed) and their underlying genetic basis (e.g., the number of genes that influence their expression) also mean that neither $\mathbf{u}$ nor $\mathbf{G}$ is necessarily constant (Steppan et al. 2002).

Equation 1 can be used to review the evolution of three traits: a male sexual display $(d)$, a female mating preference $(p)$, and residual fitness or viability $(v)$. The term $v$ is difficult to quantify empirically: It is the naturally selected fitness left over after variation in fecundity solely attributable to mate quality or number is excluded. It cannot be measured, unless assumptions are made about the function relating investment in $d$ to $v$ : By how much is $v$ reduced as $d$ increases? The implicit assumption that the value of $v$ is independent of the sex in which the relevant genes are expressed is also likely to be invalid in many cases (Fedorka \& Mousseau 2004).

Fuller et al. (2005) decompose $\beta$ into sexual selection $\left(\beta_{S}\right)$ and natural selection $\left(\beta_{N}\right)$. By Fuller et al.'s definition (which we follow here), sexual selection covers any selection that arises as a result of variation in the number and/or quality of mates. 
Direct benefits of mate choice: an increase in a fitness component (e.g., survival or fecundity) of a choosy individual that is not based on the genetic quality of offspring
The initial equation when expanded is

$$
\left(\begin{array}{c}
\Delta \bar{d} \\
\Delta \bar{p} \\
\Delta \bar{v}
\end{array}\right)=\left(\begin{array}{ccc}
V_{d} & C_{d p} & C_{d v} \\
C_{d p} & V_{p} & C_{p v} \\
C_{d v} & C_{p v} & V_{v}
\end{array}\right) \times\left(\left[\begin{array}{c}
\beta_{N d} \\
\beta_{N p} \\
\beta_{N v}
\end{array}\right]+\left[\begin{array}{c}
\beta_{S d} \\
\beta_{S p} \\
\beta_{S v}
\end{array}\right]\right)+\left(\begin{array}{l}
u_{d} \\
u_{p} \\
u_{v}
\end{array}\right) .
$$

We can now discuss the various scenarios for the evolution of a mating preference, $p$, in terms of different selective forces and how these are influenced by additive genetic variance $(V)$ in each trait and genetic covariances $(C)$ between traits.

\section{Direct Selection: The Believable Trinity of Sensory Bias, Direct Benefits, and Sexual Conflict}

The least-controversial forms of selection on mate choice occur when there is direct selection (where $\beta_{N p}$ or $\beta_{S p} \neq 0$ ) and additive genetic variation in choice $\left(V_{p}>0\right)$. When choice is favored by direct natural selection $\left(\beta_{N_{p}}>0\right)$, the resultant preference is not an adaptation to ensure chosen males confer benefits; rather it is an incidental effect of selection in another context. Ignoring mutation, at equilibrium the preference is under natural selection $\left(\beta_{N p}=0\right)$, and there is no sexual selection on it. In contrast, the display trait evolves (if $V_{d}>0$ ) until there is an equilibrium between sexual and natural selection on it $\left(\beta_{S d}=-\beta_{N d}\right)$. Sexual-selection theory refers to this process as sensory bias or sensory exploitation (Endler \& Basolo 1998). Many empirical examples have been proposed to illustrate sensory bias (e.g., Garcia \& Ramirez 2005), but, as Fuller et al. (2005) point out, critical tests are usually lacking. One key aim should be to demonstrate $\beta_{S p} \equiv 0$ by showing that the value of $d$ does not indicate whether a male confers greater benefits or reduced mating costs to females (remember that $\equiv$ means no values of the trait experience selection, as opposed to a local selective optimum having been reached). Fuller et al. (2005) suggest three empirical tests:

1. Breeding experiments to see if male phenotype predicts female reproductive performance. Many such studies show that $\left|\beta_{S p}\right|>0$ (e.g., Friberg \& Arnqvist 2003, Head et al. 2005).

2. Selection experiments in which females are either randomly assigned mates or allowed to choose (given $\beta_{N p}=0$ and $\beta_{S p} \equiv 0$, there should be no decline in the preference as there are no sexually selected costs to random mating). Again, available studies generally suggest $\left|\beta_{S p}\right|>0$ (see Hosken \& Tregenza 2005).

3. Artificial selection for a female trait that might influence mate choice (e.g., Endler et al. 2001). Because strong selection on almost any trait is likely to influence mating patterns, and hence mate choice (Wiley \& Poston 1996), the value of this approach depends critically on whether experiments mimic a plausible force of natural selection and help to understand the proximate basis of mating preferences.

Another uncontroversial way in which a mating preference can evolve is through direct sexual selection on choice $\left(\beta_{S p}>0, V_{p}>0\right)$. In standard terminology, this is defined as natural selection for direct benefits (e.g., Hoelzer 1989, Price et al. 
1993). It assumes an initially positive phenotypic correlation between a male display, $d$, and the benefit of mating with him, owing to a direct increase in female fitness or offspring survival. For example, males in better condition might provide better parental care or be less likely to transfer venereal diseases. Assuming $V_{d}>0$ and again ignoring $\mathbf{u}, d$ evolves until natural selection opposes sexual selection, $\beta_{S d}=-\beta_{N d}$. The mating preference similarly evolves under sexual selection, until the benefit of stronger preferences is countered by their costs owing to, for example, the energetics of mate searching $\left(\beta_{S p}=-\beta_{N_{p}}\right)$. In some cases, however, one can also imagine a preference that has no naturally selected $\operatorname{cost}\left(\beta_{N_{p}}=0\right)$ initially evolving because $\beta_{S p}>0$, until $\beta_{S p}=0$ because the benefit originally signaled by $d$ is eventually exactly countered by a fitness cost that $d$ itself imposes on females (e.g., long-tailed males provide less parental care) (Price et al. 1993; see also Kokko 1998b).

Sexual-conflict theory is all about the diverse range of direct costs that a male can impose on females while attempting to increase his own fitness (Arnqvist \& Rowe 2005). Let us consider a situation where mate choice has already evolved: Females prefer males with larger $d$. How do direct costs influence the subsequent evolution of the mating preference? There could be sexual selection to diminish preferences if attractive males impose greater direct costs on females than less-attractive males $\left(\beta_{S p}<0\right)$. There has long been circumstantial evidence for such costs in birds where females contribute relatively more to parental care if their mate is attractive (Møller \& Jennions 2001). Recent experimental studies report that females assigned attractive males (Head et al. 2005) or males with phenotypes correlated with attractiveness (Friberg \& Arnqvist 2003, Pitnick \& García-González 2002) can have decreased lifetime offspring production. This begs a question: If some males impose direct costs, why prefer them? Logically, one seeks out other forces counteracting the preference reduction. One possibility is that females gain genetic benefits for their offspring, which offset the direct costs these harmful males impose (Hosken \& Tregenza 2005, Kokko 2005). But what about compensatory direct benefits?

One major area of sexual conflict regards mating rates (Arnqvist \& Rowe 2005). The optimal number of matings is almost certainly lower for a female than for a male (Arnqvist \& Nilsson 2000). This can create sexual selection to elevate mating preferences $\left(\beta_{S p}>0\right)$ to reduce the number of times females mate (Gavrilets et al. 2001; for an updated review, see Rowe et al. 2005). Such cost minimization results in some males mating more often than others, owing to female traits. This is, by definition, a form of mate choice (Wiley \& Poston 1996) involving a mating preference/resistance that selects for attractive, seductive, or persistent males (Holland \& Rice 1998). If stronger mating preferences help reduce the direct cost of excessive mating, they can in principle counter the selection on females to avoid mating with males with larger values of $d$ that impose greater direct costs per mating. Although not explicitly modeled, the difference between the cost of rejecting and accepting a male could increase with $d$. This would make it cheaper to mate with preferred males (larger $d$ ) than to reject them. (Obviously some males must be cheaper to reject than accept, otherwise females would accept all males.)

There could also be selection to mate less readily under certain conditions, e.g., when predators are common so that the benefit of choosiness is a reduced mating 
G-matrix: a table of genetic variances (along the diagonal) and covariances (elsewhere) between traits rate $\left(\beta_{p}>0\right)$. This creates sexual selection on males if some traits increase the rate of encounter with females in their receptive state. Putting aside sexual conflict over mating rates and differential costs of rejection, however, current mathematical models suggest females should avoid mating with males that impose greater costs per mating unless there are genetic benefits. [We have so far ignored mutations $\mathbf{u}$, but they play a large role in this argument (Kokko 2005).]

Under sexual conflict, the equilibrium situation is still one where sexual selection to increase $p$ is countered by natural selection $\left(\beta_{S p}=-\beta_{N p}\right)$. Natural selection to reduce $p$ arises, for example, if overly resistant females take longer to acquire a mate and therefore breed at suboptimal times (e.g., Backwell \& Passmore 1996). In sum, in the above three scenarios, there is no net-directional selection on $p$ at equilibrium. This reflects opposing sexual and natural selection in sexual conflict and direct benefit models. Although sexual conflict has recently been elevated in status as an explanation for mate choice, the only real distinction between it and direct benefit models is that the main reason not to reduce $p$ is the cost of an increased mating rate. Sexual-conflict models may be seen as distinctive because they alone involve male-imposed costs. However, given direct benefits to matings with certain males, one could, without torturing the language, equally well state that $p$ is maintained through the cost of mating with males with smaller displays that provide fewer benefits. The sensory bias scenario differs from the other two, however, because there is stabilizing natural selection on $p$ at equilibrium.

\section{Indirect Selection: The Devil Is in the Details}

In the section above, aside from noting that traits only evolve if $\mathbf{V}>0$, we studiously ignored the G-matrix and mutations. In effect, we prevented a genetic covariance between traits developing. Is this realistic? Can the effect of mutations be ignored? Most importantly, if genetic correlations between traits evolve, do equilibrium states stay the same? The answers are no, no, and no.

Given additive genetic variation $\left(V_{p}>0, V_{d}>0\right)$, genes for a display and those for a preference associate more often than expected because choosy females mate more often with males with better displays. This gametic phase disequilibrium results in a positive genetic covariance $\left(C_{d p}>0\right)$ between $d$ and $p$ (Lande 1981). Whenever $p>0$, as a result of drift or sensory bias (Payne \& Pagel 2000), mate choice results in direct sexual selection on display $d\left(\beta_{S d}>0\right)$ and indirect selection on preferences $p$ because $C_{d p}>0$. As $p$ increases, so does $\beta_{S d}$; a positive feedback loop is established, driving an increase in $p$. Lande (1981) formally showed that rapidly increasing values of $p$ and $d$ evolve if $C_{d p}>V_{d}$, but if $C_{d p}<V_{d}, d$ only evolves to match $p$.

Indirect selection on $p$ is often referred to as the Fisher process and the rapid coevolution of exaggerated display and choice as the Fisherian runaway (Andersson 1994). This is in recognition of Fisher (1930) who first outlined this process in a verbal model to account for Darwin's claim that female aesthetic sensibilities drive the evolution of male courtship traits. In the absence of any naturally selected cost of $p\left(\beta_{N p} \equiv 0\right)$, a line of equilibrium emerges: For any given level of $p$, there is a matching 
expression of $d ; \beta_{S p} \equiv 0$ because all males are of equal fitness. The sexually selected benefit of a larger display is perfectly balanced by natural selection owing to the mortality cost it imposes $\left(\beta_{S d}=-\beta_{N d}\right)$. Females gain nothing by producing moreattractive sons with higher mating success, as these sons have lower survivorship. We discuss this further below to consider what happens when preferences are costly $\left(\beta_{N p} \neq 0\right)$.

Mate-choice models are most controversial when we consider the third trait of residual fitness, or viability $(v)$, that covaries positively with male display $\left(C_{d v}>0\right)$. Phenotypic viability (measured by proxies such as lifespan, immunocompetence, or physiological condition) may still not be positively correlated with sexual display (Grafen 1990a, Hunt et al. 2004b, Kokko 2001, Tomkins et al. 2004). Depending on the life-history schedule and effect of display on mating success (Kokko 1998a), males with the highest residual viability may invest disproportionately in display and end up in the worst condition with the lowest survivorship (e.g., Hunt et al. 2004a). Lifehistory schedules also determine whether display increases with age (Kokko 1998a, Proulx et al. 2002).

The most-likely cause of a positive relationship, $C_{d v}>0$, is condition-dependent expression of sexual displays (Tomkins et al. 2004, Zahavi 1977). Numerous studies have shown that sexual display is sensitive to male condition (Bonduriansky \& Rowe 2005, but see also Cotton et al. 2004). Condition refers to a male's state prior to development of the display and is therefore equivalent to residual fitness. Fuller et al. (2005) suggested that $V_{d}$ can be zero. One can indeed imagine a scenario where the gene for $d$ simply codes for condition-determined expression. Quantitative genetics has, however, its basis in partitioning phenotypic variation among genetic and environmental sources. Genes for conditions that covary with the expression of $d$ are thus synonymous with additive genetic variation in $d$. If condition is heritable, a father-son regression will show that their display sizes covary, which, excluding effects of a shared environment, is generally interpreted as $V_{d}>0$. Although not widely discussed, if condition-dependent display and viability have a near-identical genetic basis (pleiotropy), they could be considered as one trait [although whether genotype by environment interactions strongly affect allocation decisions about investment in $d$ is still undetermined (Greenfield \& Rodriguez 2004)]. How $d$ can honestly signal $v$ to females is, of course, a separate question (Getty 2006). So too is the question of what maintains additive genetic variation in $v\left(V_{v}\right)$, i.e., lek paradox (Kirkpatrick \& Ryan 1991, Rowe \& Houle 1996). We return to the role of mutations u below.

Given positive $V_{d}, V_{p}, C_{d p}$, and $C_{d v}$, there is also a genetic correlation between $p$ and $v\left(C_{p v}>0\right)$. Natural selection on viability $\left(\beta_{N v}>0\right)$ therefore generates additional indirect selection on mate choice: Choosy females produce fitter offspring and not just more-attractive sons. This is commonly referred to as the good-gene viability indicator, or handicap model of sexual selection. It was first verbally modeled by Zahavi $(1975,1977)$. After some initial failures, this process has been successfully modeled many times (e.g., Grafen 1990a, Iwasa et al. 1991, Iwasa \& Pomiankowski 1999, Pomiankowski 1987b).
Lek paradox: the problem of maintaining sufficient genetic variation in a trait subject to directional natural and sexual selection to sustain costly nonrandom mating 
Indirect benefits of mate choice: an increase in the fitness of a choosy individual based on the genetic quality of offspring

\section{Indirect Selection: The Difficult Second Album}

The role of indirect selection in the evolution of mate choice has sparked considerable debate. First, many questioned whether certain processes could work in principle [e.g., compare Kirkpatrick (1986) with Grafen (1990b) debating Zahavis (1975) handicap ideas]. Usually differences arose because models made different structural assumptions, for example, about the relationship between the expression of genes for $v$ and $d$ (see Eshel et al. 2000, 2002), or violated assumptions implicit in other models, for example, the assumption that $V_{v}$ remains positive. In other cases, minor modifications to the original models were required. For example, early models of the Fisher process concluded that costly preferences cannot be maintained (e.g., Kirkpatrick 1985), but subsequent work showed this no longer applies if there is biased mutation on a complex male display (Pomiankowski et al. 1991). Mutations u can therefore make a qualitative difference, even in (by far the most-common type of) models that ignore their effect on the G-matrix and therefore do not directly help answer the question of how $\mathbf{V}>0$ can be maintained.

Second, many question the importance of indirect selection as a major force. Indirect benefits of mate choice are probably small. A chain of events are required for choosy females to obtain genetic benefits (Kirkpatrick \& Barton 1997), so the rewards are minimal if even one process does not work as required. For example, erosion of additive genetic variation in fitness (lower $V_{v}$ ), inaccurate choice (lower $C_{d p}$ ), unreliable signals of viability (lower $C_{d v}$ ), and weaker sexual selection on male display (lower $\beta_{S d}$ ) all reduce the benefits of choosiness. The key empirical question, therefore, becomes, how strong is direct selection on choosiness? If it is weak, then small, indirect genetic benefits may still suffice to maintain preferences that, by virtue of being cheap to express, have significant consequences on trait evolution in the opposite sex. Unfortunately, the costs of choosiness are largely unknown (Houle \& Kondrashov 2002), although some researchers have recently attempted to compare the relative strength of direct costs and indirect benefits (Arnqvist \& Kirkpatrick 2005), as well as to quantify precisely mate-sampling costs (Byers et al. 2005).

Third, confusion exists about whether different processes should or can be distinguished. Specifically, there is a long history of trying to dichotomize traits into those that evolve or are maintained solely through Fisherian or good-genes processes. This has led to a quixotic attempt to empirically test these as alternatives, generating controversy where none exists. In the section below, we outline why we believe there is a unitary process that can be accommodated in a single set of models. This view is neither new nor ours alone. To quote Pomiankowski \& Iwasa (2001), "[t]he two hypotheses are really extremes on a continuum... In the real world, male traits involved in mate choice simply do not fall into two types, Fisher and handicap traits" (p. 216).

\section{A UNITARY MODEL OF INDIRECT BENEFITS}

We have recently been involved in debating whether processes for the evolution of mate choice that rely on benefits from producing attractive sons are fundamentally 
different from those from producing more-viable offspring (Cameron et al. 2003, Eshel et al. 2000, Fuller et al. 2005, Kokko et al. 2002). We first outline the arguments others offer for such a division before presenting the case for a unified view. As a modeling exercise, one can certainly eliminate certain indirect benefits to clarify an argument, in the same way as a researcher controls for confounding factors known to influence the outcome of an experiment. On the basis of such work, we know, for example, that the handicap process works even if choosy females do not produce sons with higher mating success (Grafen 1990a,b).

It is surprisingly difficult to find an unambiguous definition of the Fisher process in the literature. One criterion is that choosy females produce more-attractive sons owing to linkage disequilibrium $\left(C_{d p}\right)$ (e.g., Fuller et al. 2005, Mead \& Arnold 2004). Some authors focus on self-reinforcing selection on $p$ and $d$ (e.g., Heisler et al. 1987, Takahashi 1997) or, more narrowly, on the possibility of open-ended runaway (Fisher's runaway process sensu Iwasa \& Pomiankowski 1995 and Day 2000). Others provide a definition where the only forces on male display are natural selection for reduction and female choice for exaggeration (e.g., van Doorn et al. 2004; implicit in Shuster $\&$ Wade 2003, p. 185). All these features arise in a classical Fisherian model such as Lande (1981), but which are essential?

Linkage disequilibrium, self-reinforcement, and runaway cannot all define the Fisher process as they do not always co-occur. Linkage disequilibrium, per se, does not always predict self-reinforcing selection as the male trait does not evolve if $p$ is below a critical threshold value (Pomiankowski 1988) and runaway only occurs if $C_{d p}>V_{d}$ (Lande 1981). What of the requirement that there is only natural selection against and sexual selection for the male display? Reading Fisher's (1930) original contribution provides some insight. He allowed a positive correlation between a male trait and viability to initiate the spread of the female preference, although the relationship eventually turned negative during the coevolutionary process.

Following Fisher's lead (see also Kirkpatrick \& Ryan 1991), here we define the Fisher process as simply referring to cases where female preferences experience indirect selection caused by offspring inheriting their father's attractiveness (the so-called sexy sons). There is no reference to any correlation between male display and other fitness traits, although models that address the Fisher process on its own usually analyze cases where male display reduces survival (Lande 1981). There is also no statement about whether choice is costly. Weatherhead \& Robertson (1979) originally used the term sexy son in a context of costly choice, but the term is now more widely applied.

Cameron et al. (2003) argue that we must formally distinguish Fisherian and good-genes processes because a costly female preference can never be maintained solely by the indirect benefit of producing sexy sons (see also Kirkpatrick \& Ryan 1991, Pomiankowski 1987a). Their model shows that equilibrium is only reached when female choice and male display are at their naturally selected optima. Thus the Fisher process is unstable and will not be seen in nature. As Pomiankowski et al. (1991) first noted, however, $p$ and $d$ lie above their naturally selected optimum at equilibrium if there is a negative mutational bias on the male trait. Cameron et al.'s (2003) insight is that in the absence of female choice, deleterious mutations push $d$ below its naturally selected optimum. This implies that natural selection favors males 
Fisher-Zahavi process: coevolution of female preferences and male displays influenced by the fact that females mating with preferred fathers produce sexy sons with larger ornaments. As attractive males are more viable at this stage, their evolution cannot be considered a Fisher process, but it is rather a version of good genes.

Our interpretation differs for the following reason: One must distinguish between a process and a model that exemplifies its evolution. Of necessity, every model makes various assumptions that typically make it one of many possible examples of how a process might run. Relaxing model assumptions one by one is why we usually find many published models addressing the same topic. Cameron et al. (2003) make two important assumptions: (a) The male display $d$ can take on any value, positive or negative, and ( $b$ ) mutation pressure is constant and negative for all values of $d$. What happens if we change these assumptions and consider a male trait that can only have non-negative values (e.g., crest height) with a naturally selected optimum at $d=0$ (absence of crest)? This simple assumption yields a model with an equilibrium where female choice is costly and the male trait is subject to a destructive mutational bias (i.e., mutations tend to interfere negatively with the development of a complex ornament). However, in the absence of female choice, the net mutation bias is necessarily positive: Traces of an ornament that is not selected for can be created by mutation, thus $d>$ 0 (see Supplemental Appendix; follow the Supplemental Material link from the Annual Reviews home page at http://www.annualreviews.org).

This example is neither more nor less general than that of Cameron et al. (2003). Our model differs because it considers a case where natural selection only ever favors smaller traits, and consequently natural and sexual selection on $d$ are never in the same direction. If the Fisher process cannot lead to trait exaggeration unless elements of good genes are added, this example of a stable costly preference at equilibrium should be impossible.

The issue reduces to one of definitions. Many models investigate the Fisher process when there is mutational input or display is positively correlated with viability-related fitness (e.g., Eshel et al. 2000, Kokko et al. 2002, Pomiankowski et al. 1991). Cameron et al. (2003) appear to limit the definition of a Fisher process to a purer context where these factors are excluded. It is illustrative to compare the implicit definition of Cameron et al. (2003) with that of Shuster \& Wade (2003, pp. 182-85). Both envisage a female preference for a trait that is originally related to viability but no longer is after preference evolution. The interpretations are exact opposites of each other: Shuster \& Wade use the same example to reject the idea of good genes in favor of Fisher. Definitions cannot be imposed, but we recommend the general usage and consider a model to incorporate the Fisher process if it helps to delimit the conditions under which the production of sexy sons influences female-choice evolution (Parker 2006). The term Fisher-Zahavi process appears appropriate when additionally emphasizing the possibility that male displays covary with viability-related traits (see Eshel et al. 2000).

At equilibrium, there is always a choice among males who offer different indirect and/or direct benefits. The net effect of mating with a male can be quantified as the sum of its offsprings' reproductive values, including attractiveness of male offspring (Kokko et al. 2002, McNamara et al. 2003). This argument has been incorrectly criticized for ignoring $C_{d p}$ (Fuller et al. 2005); if this critique were true, the method could not conceivably produce cases where females prefer less-viable males yet preferences 
still spread (Kokko et al. 2002). Reproductive-value calculations include $C_{d p}$ implicitly, as choosy females are more likely to mate with a certain subset of males in each generation. Reproductive value also depends on the environment. Small changes in environmental conditions can lead to different phenotypic relationships between major fitness traits, such as display and longevity, without any change in the underlying genetic architecture (Kokko et al. 2002). This is because male attractiveness is often subject to life-history trade-offs (Grafen 1990b, Kokko 2001), and males can adaptively adjust their current investment in attractiveness in response to environmental challenges (Badyaev \& Qvarnström 2002, Candolin 2000, Hunt et al. 2004a).

It might be impossible to distinguish empirically between a pure Fisher process and cases where display and residual fitness $v$ are genetically correlated. A partial solution is to look only at the viability of daughters, but this assumes that the fitness effects of genes are not sex-dependent, although recent work suggests otherwise (Chippindale et al. 2001, Fedorka \& Mousseau 2004). This is one purely pragmatic reason why the Fisher process (a benefit of producing sexy sons, usually because $C_{d p}>0$ ) and the effects of a correlation between male display and other fitness-related traits $\left(C_{d v}>0\right)$ are best seen as part of a continuum of outcomes of a unitary evolutionary process (Kokko et al. 2002, Pomiankowski \& Iwasa 2001; compare Brooks 2000 and Kokko 2001).

\section{ON PHENOTYPIC AND QUANTITATIVE GENETIC MODELS}

Sometimes the conclusions of different sexual-selection models seem related to the modeling technique used. Fuller et al. (2005), Shuster \& Wade (2003) and others have claimed that phenotypic models are inappropriate to model aspects of sexual selection that depend on genetic covariances. This is an unfortunate criticism: Methods that appear to have ignored a term may simply have let it emerge from the model, rather than giving it a fixed value. Phenotypic models preferentially emphasize that mate choice changes the types of offspring produced (Kokko et al. 2002, McNamara et al. 2003), instead of assuming a fixed $C_{d p}>0$.

Models can differ in the simplifying assumptions made, but they are, ultimately, special cases of the same mathematical framework (Hammerstein 1996, Page \& Nowak 2002, Waxman \& Gavrilets 2005). The same assumptions (e.g., biased mutation) are expressed in different ways depending on the modeling technique. The apparent effect of modeling techniques on conclusions only arises because researchers using different approaches tend to make different assumptions. All models are approximations. We know, for example, that the G-matrix must ultimately evolve in response to mutations $\mathbf{u}$, but this is typically ignored in quantitative genetic models. Empiricists prefer to ignore u's immediate effect as well, as the G-matrix is easier to estimate assuming $\mathbf{u}=0$. This is convenient, but because $\mathbf{u}$ is important for long-term evolution (Iwasa et al. 1991, Pomiankowski et al. 1991), the outcome may feature a mismatch between short- and long-term evolutionary arguments (Pigliucci \& Schlichting 1997). We prefer the current plurality in modeling, as each modeling tool has some disadvantages, making it more difficult to capture some features of reality than others. Using one approach can lead only to the neglect of some real-world 
issues. To quote the psychologist Abraham Maslow, "[i]f the only tool you have is a hammer, you tend to see every problem as a nail."

\section{IDEAS ON TESTING THESE IDEAS}

The prediction that choosy females produce sexy sons is critical to all models of indirect selection on choice. If inheritance is genetic, $C_{d p}>0$, although genetic preferences for displays transmitted culturally from father to son can evolve in principle (Ihara et al. 2003, McNamara et al. 2003). Several empirical studies have used artificial selection on $d$ to show that $p$ evolves as a correlated response (e.g., Brooks \& Couldridge 1999, Wilkinson \& Reillo 1994), but results are difficult to interpret. $C_{d p}$ is maintained by nonrandom mating, so it should break down rapidly during artificial selection using forced random matings, yet correlated responses to selection may occur owing to sexual selection within selected lines in which mate choice can occur (Gray \& Cade 1999).

Within-generation estimates of $C_{d p}$ are valuable (e.g., Ritchie et al. 2005). We suspect future estimates will show that at equilibrium $C_{d p}$ is small both in species where mate choice is strong and unconstrained and in species where females can rarely exercise their preferences freely. For example, the unanimity of choice in lekking birds means there is little phenotypic variation in choice, and thus little scope for current additive genetic variation. Similarly, the requirement of social monogamy in most passerines means that many females mate with an available male rather than the one they would prefer, so $d$ and $p$ (measured as observed choice) barely covary (Qvarnström et al. 2006). Blows' (1999) study of the evolution of $C_{d p}$ after experimental hybridization of Drosophila serrata and D. birchii illustrates our point. Hybridization disrupted the linkage disequilibrium underlying $C_{d p}$, resulting in a relatively small correlation of 0.39 at generation 5. Choice built the correlation up to 1.02 at generation 37 , but $C_{d p}$ then plunged to -0.04 by generation 56 , possibly because much of the variation in choice and display present at and before generation 37 had been fixed by generation 56 .

A conceptual issue emerges: Must we only consider the extant set of females when calculating $C_{d p}$ ? Let us assume all peahens prefer the peacock with the largest train; hence $C_{d p}=0$. There is no Fisher process evident, but $C_{d p}>0$ is reintroduced if a mutation causes a peahen to mate randomly. As her sons are unsuccessful, this mutant soon is eliminated. Should we not distinguish between cases where $C_{d p}=0$ because females show a near-unanimous preference for the sexiest males and a case where $C_{d p}=0$ because females mate randomly with respect to the value of $d$ ? This highlights the difficulty of arguing about long-term evolution using short-term measures of selection (Hammerstein 1996, Pigliucci \& Schlichting 1997). Empirical progress clearly requires a combination of single-generation, multigeneration, and clever experimental approaches.

The most-fundamental tests of mate-choice models are whether $(a)$ female choice maximizes the sum of direct and indirect benefits; $(b)$ direct selection results in females choosing males that do not maximize their fitness when compared with other mates, but this behavior is still adaptive because the mechanism driving choice maximizes 
net-female fitness (e.g., by reducing the cost of excess mating); or $(c)$ female choice is maladaptive (Gustafsson \& Qvarnström 2006). The extent to which (b) occurs may depend on how easily females can modify the effect of traits valuable in a nonmating context that might adversely influence mate choice (Houle \& Kondrashov 2002) or on whether females can make themselves unavailable to males by becoming sexually unreceptive (Rowe et al. 2005). In turn, $(c)$ is more likely if male-female coevolution is at a stage where the necessary mutations have not arisen for females to adjust their behavior to maximize their fitness, given the male types to which they are currently exposed. Empirical tests of these scenarios are daunting (Hunt et al. 2004b). It is difficult to produce an all-inclusive measure of offspring fitness and merge it with parental-lifetime reproductive success, and even this is only a proxy for fitness (Brommer et al. 2004).

\section{What We Tend to Forget to Measure}

To summarize, all models agree that mate choice can evolve by a combination of direct and/or indirect benefits. In the latter case, the selection on female preferences is weak and easily overridden by the costs of choice. It is important to remember, however, that even small changes in female behavior (which cost little) can generate strong selection when a male's fitness depends primarily on his mating success (Alatalo et al. 1998, Reynolds \& Gross 1990).

There is an important way to phrase this prediction that is rarely used in the literature: Preferences can exist despite substantial costs of expression if they yield large direct benefits, whereas preferences that evolve in the absence of direct benefits should be cheap to express (unless there is substantial genetic variation in overall fitness). Although we do not discuss the lek paradox extensively, new multivariate methods indicate it is far from resolved (see Blows \& Hoffmann 2005). In the case of a practically cost-free behavior that creates mating biases, the consequences for maletrait evolution can still be dramatic. Unfortunately, showing there are no measurable costs in the appropriate life-history currency is probably even more demanding than measuring indirect benefits. It may, however, still be possible to rank preferences in terms of their estimated costs and to test the prediction that costs are smaller where we presume only indirect benefits than in cases where direct benefits of choice have been firmly established. The challenge is there.

\section{SUMMARY POINTS}

1. The multitude of mathematical models in the field of sexual selection can appear confusing, but the diversity of models, similar to the diversity of experimental approaches, is necessary for healthy progress toward understanding which assumptions are crucial for an argument. There is a growing trend toward more-inclusive models that make fewer implicit assumptions and avoid inconsistencies generated by too many fixed parameters. 
2. The importance of self-consistent modeling is highlighted by recent reassessment of the way parental sex roles follow from anisogamy. Earlier explanations based on PRRs are at odds with the fact that total reproduction by the male population cannot exceed that by the female population. Instead, parental roles can follow from anisogamy because it causes sperm competition and sexual selection, which feed back to the evolution of parental roles.

3. Mate-choice evolution can be influenced by direct and indirect benefits. These can all be merged into a simple, quantitative genetic framework, including cases in which superfluous mating is costly to females. Care should be taken, however, when arguing about long-term evolution using shortterm measures of selection.

4. The recent debate regarding the distinctness of the Fisher process from the good-genes hypothesis boils down to definitions. We suggest using the term Fisher-Zahavi process to help remember that indirect benefits can include both improved viability and greater attractiveness of offspring.

5. Detecting indirect benefits is often challenging as the lek paradox (in its univariate or multivariate form) predicts these to be small; however, costs of expressing preferences can be minute too. A central prediction emerges that requires future tests: Costs of preference expression should be small in cases of indirect benefits, whereas they can be larger if direct benefits are involved.

\section{ACKNOWLEDGMENTS}

Many people have helped us to formulate our ideas more clearly, and we are particularly indebted to Göran Arnqvist, Pat Backwell, Troy Day, Matt Hall, David Houle, John McNamara, Locke Rowe, and Geoff Parker; we would like to add many others. The frank comment by a Larus sp. individual in Toronto must also be mentioned here. Funding was provided by the Academy of Finland and by the Australian Research Council.

\section{LITERATURE CITED}

Agrawal AF. 2001. Sexual selection and the maintenance of sexual reproduction. Nature 411:692-95

Alatalo RV, Kotiaho J, Mappes J, Parri S. 1998. Mate choice for offspring performance: major benefits or minor costs? Proc. R. Soc. London Ser. B 265:2297301

Alonzo SH, Warner RR. 2000. Female choice, conflict between the sexes and the evolution of male alternative reproductive behaviours. Evol. Ecol. Res. 2:14970

Andersson M. 1994. Sexual Selection. Princeton, NJ: Princeton Univ. Press 
Arnqvist G, Kirkpatrick M. 2005. The evolution of infidelity in socially monogamous passerines: the strength of direct and indirect selection on extrapair copulation behaviour in females. Am. Nat. 165:S26-37

Arnqvist G, Nilsson T. 2000. The evolution of polyandry: multiple mating and female fitness in insects. Anim. Behav. 60:145-64

Arnqvist G, Rowe L. 2005. Sexual Conflict. Princeton, NJ: Princeton Univ. Press

Backwell PRY, Passmore NI. 1996. Time constraints and multiple choice criteria in the sampling behaviour and mate choice of the fiddler crab, Uca annulipes. Behav. Ecol. Sociobiol. 38:407-16

Badyaev AV, Qvarnström A. 2002. Putting sexual traits into the context of an organism: a life-history perspective in studies of sexual selection. Auk 119:301-10

Barlow GW. 2005. How do we decide that a species is sex-role reversed? Q. Rev. Biol. 80:28-35

Berglund A, Rosenqvist G. 2003. Sex role reversal in pipefish. Adv. Study Behav. 32:131-67

Blows MW. 1999. Evolution of the genetic covariance between male and female components of mate recognition: an experimental test. Proc. R. Soc. London Ser. B 266:2169-74

Blows MW, Hoffmann AA. 2005. A reassessment of genetic limits to evolutionary change. Ecology 86:1371-84

Bonduriansky R, Rowe L. 2005. Sexual selection, genetic architecture, and the condition dependence of body shape in the sexually dimorphic fly Prochyliza xanthostoma (Piophilidae). Evolution 59:138-51

Brommer JE, Gustafsson L, Pietiäinen H, MeriläJ. 2004. Single-generation estimates of individual fitness as proxies for long-term genetic contribution. Am. Nat. 163:505-17

Brooks R. 2000. Negative genetic correlation between male sexual attractiveness and survival. Nature 406:67-70

Brooks R, Couldridge V. 1999. Multiple sexual ornaments coevolve with multiple mating preferences. Am. Nat. 154:37-45

Bulmer MG, Parker GA. 2002. The evolution of anisogamy: a game-theoretic approach. Proc. R. Soc. London Ser. B 269:2381-88

Byers JA, Wiseman PA, Jones L, Roffe TJ. 2005. A large cost of female mate sampling in pronghorn. Am. Nat. 166:661-68

Byrne PG, Simmons LW, Roberts JD. 2003. Sperm competition and the evolution of gamete morphology in frogs. Proc. R. Soc. London Ser. B 270:2079-86

Cameron E, Day T, Rowe R. 2003. Sexual conflict and indirect benefits. F. Evol. Biol. 16:1055-60

Candolin U. 2000. Changes in expression and honesty of sexual signalling over the reproductive lifetime of sticklebacks. Proc. R. Soc. London Ser. B 267:2425-30

Chippindale AK, Gibson JR, Rice WR. 2001. Negative genetic correlation for adult fitness between sexes reveals ontogenetic conflict in Drosophila. Proc. Natl. Acad. Sci. USA 98:1671-75

Clutton-Brock TH, Parker GA. 1992. Potential reproductive rates and the operation of sexual selection. Q. Rev. Biol. 67:437-56 
Cotton S, Fowler K, Pomiankowski A. 2004. Do sexual ornaments demonstrate heightened condition-dependent expression as predicted by the handicap hypothesis? Proc. R. Soc. London Ser. B 271:771-83

Day T. 2000. Sexual selection and the evolution of costly female preferences: spatial effects. Evolution 54:715-30

Eberhard WG. 2005. Evolutionary conflicts of interest: Are female sexual decisions different? Am. Nat. 165:S19-25

Emlen ST, Oring LW. 1977. Ecology, sexual selection, and the evolution of mating systems. Science 197:215-23

Endler JA, Basolo A. 1998. Sensory ecology, receiver biases and sexual selection. Trends Ecol. Evol. 13:415-20

Endler JA, Basolo A, Glowacki S, Zerr J. 2001. Variation in response to artifical selection for light sensitivity in guppies, Poecilia reticulata. Am. Nat. 158:36-48

Eshel I, Sansone E, Jacobs F. 2002. A long-term genetic model for the evolution of sexual preference: the theories of Fisher and Zahavi re-examined. F. Math. Biol. 45:1-21

Eshel I, Volovik I, Sansone E. 2000. On Fisher-Zahavi's handicapped sexy son. Evol. Ecol. Res. 2:509-23

Fedorka KM, Mousseau TA. 2004. Female mating bias results in conflicting sexspecific offspring fitness. Nature 429:65-67

Fisher RA. 1930. The Genetical Theory of Natural Selection. Oxford, UK: Oxford Univ. Press

Forsgren E, Amundsen T, Borg AA, Bjelvenmark J. 2004. Unusually dynamic sex roles in a fish. Nature 429:551-54

Friberg U, Arnqvist G. 2003. Fitness effects of female mate choice: Preferred males are detrimental for Drosophila melanogaster females. F. Evol. Biol. 16:797-811

Fuller RC, Houle D, Travis J. 2005. Sensory bias as an explanation for the evolution of mate preferences. Am. Nat. 166:437-46

Garcia CM, Ramirez E. 2005. Evidence that sensory traps can evolve into honest signals. Nature 434:501-5

Gavrilets S, Arnqvist G, Friberg U. 2001. The evolution of female mate choice by sexual conflict. Proc. R. Soc. London Ser. B 268:531-39

Getty T. 2006. Sexually selected signals are not similar to sports handicaps. Trends Ecol. Evol. 21:83-88

Grafen A. 1990a. Biological signals as handicaps. F. Theor. Biol. 144:517-46

Grafen A. 1990b. Sexual selection unhandicapped by the Fisher process. F. Theor. Biol. 144:473-516

Gray DA, Cade WH. 1999. Correlated-response-to-selection experiments designed to test for a genetic correlation between female preferences and male traits yield biased results. Anim. Behav. 58:1325-27

Greenfield MD, Rodriguez RL. 2004. Genotype-environment interactions and the reliability of mating signals. Anim. Behav. 68:1461-68

Gustafsson L, Qvarnström A. 2006. A test of the "sexy son" hypothesis: sons of polygynous collared flycatchers do not inherit their father's mating status. Am. Nat. 167:297-302 
Hammerstein P. 1996. Darwinian adaptation, population genetics and the streetcar theory of evolution. 7. Math. Biol. 34:511-32

Härdling R, Kokko H, Arnold KE. 2003. Dynamics of the caring family. Am. Nat. 161:395-412

Head ML, Hunt J, Jennions MD, Brooks RC. 2005. The indirect benefits of mating with attractive males outweigh the direct costs. PLoS Biol. 3:289-94

Heisler L, Andersson M, Arnold SJ, Boake CR, Borgia G, et al. 1987. Evolution of mating preferences and sexually selected traits. In Sexual Selection: Testing the Alternatives, ed.JW Bradbury, M Andersson, pp. 97-118. Chichester, UK: Wiley

Hoelzer GA. 1989. The good parent process of sexual selection. Anim. Behav. 38:1067-78

Holland B, Rice WR. 1998. Perspective: chase-away sexual selection: antagonistic seduction versus resistance. Evolution 52:1-7

Hosken DJ, Tregenza T. 2005. Evolution: Do bad husbands make good fathers? Curr. Biol. 15:836-38

Houle D, Kondrashov AS. 2002. Coevolution of costly mate choice and conditiondependent display of good genes. Proc. R. Soc. London Ser. B 269:97-104

Houle D, Morikawa B, Lynch M. 1996. Comparing mutational variabilities. Genetics 143:1467-83

Houston AI, McNamara JM. 2002. A self-consistent approach to paternity and parental effort. Philos. Trans. R. Soc. London Ser. B 357:351-62

Houston AI, McNamara JM. 2005. John Maynard Smith and the importance of consistency in evolutionary game theory. Biol. Philos. 20:933-50

Houston AI, Szekely T, McNamara JM. 2005. Conflict between parents over care. Trends Ecol. Evol. 20:33-38

Hunt J, Brooks R, Jennions MD, Smith MJ, Bentsen CL, Bussière LF. 2004a. Highquality male field crickets invest heavily in sexual display but die young. Nature 432:1024-27

Hunt J, Bussière LF, Jennions MD, Brooks R. 2004b. What is genetic quality? Trends Ecol. Evol. 19:329-33

Ihara Y, Aoki K, Feldman MW. 2003. Runaway sexual selection with paternal transmission of the male trait and gene-culture determination of the female preference. Theor. Popul. Biol. 63:53-62

Iwasa Y, Pomiankowski A. 1995. Continual change in mate preferences. Nature 377:420-22

Iwasa Y, Pomiankowski A. 1999. Good parent and good genes models of handicap evolution. 7. Theor. Biol. 200:97-109

Iwasa Y, Pomiankowski A, Nee S. 1991. The evolution of costly mate preferences. II. The "handicap" principle. Evolution 45:1431-42

Jennions MD, Petrie M. 2000. Why do females mate multiply? A review of the genetic benefits. Biol. Rev. 75:21-64

Johnstone RA, Reynolds JD, Deutsch JC. 1996. Mutual mate choice and sex differences in choosiness. Evolution 50:1382-91

Kirkpatrick M. 1985. Evolution of female choice and male parental investment in polygynous species: the demise of the sexy son. Am. Nat. 125:788-810 
Kirkpatrick M. 1986. The handicap mechanism of sexual selection does not work. Am. Nat. 127:222-40

Kirkpatrick M, Barton NH. 1997. The strength of indirect selection on female mating preferences. Proc. Natl. Acad. Sci. USA 94:1282-86

Kirkpatrick M, Ryan MJ. 1991. The evolution of mating preferences and the paradox of the lek. Nature 350:33-38

Kokko H. 1998a. Good genes, old age and life history trade-offs. Evol. Ecol. 12:739-50

Kokko H. 1998b. Should advertising parental care be honest? Proc. R. Soc. London Ser. B 265:1871-78

Kokko H. 2001. Fisherian and 'good genes' benefits of mate choice: how (not) to distinguish between them. Ecol. Lett. 4:322-26

Kokko H. 2005. Treat 'em mean, keep 'em (sometimes) keen: evolution of female preferences for dominant and coercive males. Evol. Ecol. 19:123-35

Kokko H, Brooks R, McNamara JM, Houston AI. 2002. The sexual selection continuum. Proc. R. Soc. London Ser. B 269:1331-40

Kokko H, Jennions M. 2003. It takes two to tango. Trends Ecol. Evol. 18:103-4

Kokko H, Johnstone RA. 2002. Why is mutual mate choice not the norm? Operational sex ratios, sex roles, and the evolution of sexually dimorphic and monomorphic signalling. Philos. Trans. R. Soc. London Ser. B 357:319-30

Kokko H, Monaghan P. 2001. Predicting the direction of sexual selection. Ecol. Lett. 4:159-65

Lande R. 1979. Quantitative genetical analysis of multivariate evolution, applied to brain:body size allometry. Evolution 33:402-16

Lande R. 1981. Models of speciation by sexual selection on polygenic traits. Proc. Natl. Acad. Sci. USA 78:3721-25

Lande R, Arnold SJ. 1983. The measurement of selection on correlated characters. Evolution 37:1210-26

Levitan DR. 2004. Density-dependent sexual selection in external fertilizers: variances in male and female fertilization success along the continuum from sperm limitation to sexual conflict in the sea urchin Strongylocentrotus franciscanus. Am. Nat. 164:298-309

Maynard Smith J. 1977. Parental investment: a prospective analysis. Anim. Behav. 25:1-9

McNamara JM, Gasson CE, Houston AI. 1999. Incorporating rules for responding into evolutionary games. Nature 401:368-71

McNamara JM, Houston AI, Marques dos Santos M, Kokko H, Brooks R. 2003. Quantifying male attractiveness. Proc. R. Soc. London Ser. B 270:1925-32

Mead LS, Arnold SJ. 2004. Quantitative genetic models of sexual selection. Trends Ecol. Evol. 19:264-71

Møller AP, Jennions MD. 2001. How important are direct fitness benefits of sexual selection? Naturwissenschaften 88:401-15

Owens IPF, Thompson DBA. 1994. Sex-differences, sex-ratios and sex-roles. Proc. R. Soc. London Ser. B 258:93-99

Page KM, Nowak MA. 2002. Unifying evolutionary dynamics. F. Theor. Biol. 219:9398 
Parker GA. 2006. Sexual conflict over mating and fertilization: an overview. Philos. Trans. R. Soc. London Ser. B 361:235-59

Parker GA, Simmons LW. 1996. Parental investment and the control of sexual selection: predicting the direction of sexual competition. Proc. R. Soc. London Ser. B 263:315-21

Parker GA, Smith VGF, Baker RR. 1972. The origin and evolution of gamete dimorphism and the male-female phenomenon. F. Theor. Biol. 36:181-98

Payne RJH, Pagel M. 2000. Inferring the origins of state-dependent courtship traits. Am. Nat. 157:42-50

Pigliucci M, Schlichting CD. 1997. On the limits of quantitative genetics for the study of phenotypic evolution. Acta Biotheor. 45:143-60

Pitnick S, García-González F. 2002. Harm to females increases with male body size in Drosophila melanogaster. Proc. R. Soc. London Ser. B 269:1821-28

Pomiankowski A. 1987a. The costs of choice in sexual selection. 7. Theor. Biol. 128:195-218

Pomiankowski A. 1987b. The 'handicap principle' does work-sometimes. Proc. $R$. Soc. London Ser. B 127:123-45

Pomiankowski A. 1988. The evolution of female mate preferences for mate genetic quality. Oxford Surv. Evol. Biol. 5:136-84

Pomiankowski A, Iwasa Y. 2001. How does mate choice contribute to exaggeration and diversity in sexual characters? In Economics in Nature: Social Dilemmas, Mate Choice and Biological Markets, ed. R Noë, J van Hooff, P Hammerstein, pp. 20320. Cambridge, UK: Cambridge Univ. Press

Pomiankowski A, Iwasa Y, Nee S. 1991. The evolution of costly mate preferences. 1. Fisher and biased mutation. Evolution 45:1422-30

Price T, Schluter D, Heckman NE. 1993. Sexual selection when the female directly benefits. Biol. 7. Linn. Soc. London 48:187-211

Proulx SR, Day T, Rowe L. 2002. Older males signal more reliably. Proc. R. Soc. London Ser. B 269:2291-99

Puurtinen M, Kaitala V. 2002. Mate-search efficiency can determine the evolution of separate sexes and the stability of hermaphroditism in animals. Am. Nat. 160:64560

Queller DC. 1997. Why do females care more than males? Proc. R. Soc. London Ser. B 264:1555-57

Qvarnström A, Brommer JE, Gustafsson L. 2006. Testing the genetics underlying the co-evolution of mate choice and ornament in the wild. Nature 441:84-86

Randerson JP, Hurst LD. 2001. The uncertain evolution of the sexes. Trends Ecol. Evol. 16:571-79

Reynolds JD, Gross MR. 1990. Costs and benefits of female mate choice: Is there a lek paradox? Am. Nat. 136:230-43

Rifkin SA, Houle D, Kim J, White KP. 2005. A mutation accumulation assay reveals a broad capacity for rapid evolution of gene expression. Nature 438:220-23

Ritchie MG, Saarikettu M, Hoikkala A. 2005. Variation, but no covariance, in female preference functions and male song in a natural population of Drosophila montana. Anim. Behav. 70:849-54 
Rowe L, Cameron E, Day T. 2005. Escalation, retreat, and female indifference as alternative outcomes of sexually antagonistic coevolution. Am. Nat. 165:S5-18

Rowe L, Houle D. 1996. The lek paradox and the capture of genetic variance by condition dependent traits. Proc. R. Soc. London Ser. B 263:1415-21

Sheldon BC. 2002. Relating paternity to parental care. Philos. Trans. R. Soc. London Ser. B 357:341-50

Shuster SM, Wade MJ. 2003. Mating Systems and Strategies. Princeton, NJ: Princeton Univ. Press

Siller S. 2001. Sexual selection and the maintenance of sex. Nature 411:689-92

Simmons LW. 2001. Sperm Competition and Its Evolutionary Consequences in the Insects. Princeton, NJ: Princeton Univ. Press

Simmons LW, Kvarnemo C. 2006. Costs of breeding and their effects on the direction of sexual selection. Proc. R. Soc. London Ser. B. 273:465-70

Steppan SJ, Phillips PC, Houle D. 2002. Comparative quantitative genetics: evolution of the G matrix. Trends Ecol. Evol. 17:320-27

Takahasi K. 1997. Models of selective mating and the initiation of the Fisherian process. Proc. R. Soc. London Ser. B 264:839-44

Tallamy DW. 2005. Egg dumping in insects. Annu. Rev. Entomol. 50:347-70

Tomkins J, Radwan J, Kotiaho JS, Tregenza T. 2004. Genic capture and resolving the lek paradox. Trends Ecol. Evol. 19:323-28

Trivers R. 1972. Parental investment and sexual selection. In Sexual Selection and the Descent of Man, ed. B Campbell, pp. 136-79. Chicago: Aldine

van Doorn GS, Dieckmann U, Weissing FJ. 2004. Sympatric speciation by sexual selection: a critical reevaluation. Am. Nat. 163:709-25

Wade MJ, Shuster SM. 2002. The evolution of parental care in the context of sexual selection: a critical reassessment of parental investment theory. Am. Nat. 160:285-92

Waxman D, Gavrilets S. 2005. 20 questions on adaptive dynamics. 7. Evol. Biol. 18:1139-54

Weatherhead PJ, Robertson RJ. 1979. Offspring quality and the polygyny threshold: "the sexy son hypothesis." Am. Nat. 113:201-8

WebbJN, Houston AI, McNamara JM, Székely T. 1999. Multiple patterns of parental care. Anim. Behav. 58:983-93

Wiley RH, Poston J. 1996. Indirect mate choice, competition for mates, and coevolution of the sexes. Evolution 50:1371-81

Wilkinson GS, Reillo PR. 1994. Female choice response to artificial selection on an exaggerated male trait in a stalk-eyed fly. Proc. R. Soc. London Ser. B 255:1-6

Williams GC. 1966. Adaptation and Natural Selection: A Critique of Some Current Thought. Princeton, NJ: Princeton Univ. Press

Zahavi A. 1975. Mate selection-a selection for the handicap. F. Theor. Biol. 53:205-14

Zahavi A. 1977. The cost of honesty (further remarks on the handicap principle). $\mathcal{F}$. Theor. Biol. 67:603-5 


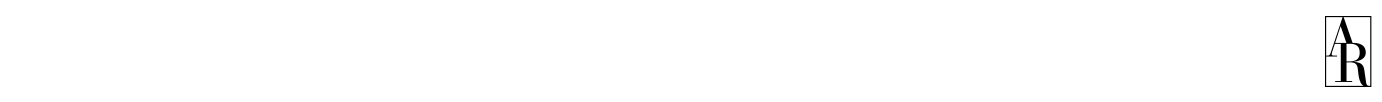

\section{Contents}

Annual Review of

Ecology, Evolution, and Systematics

Volume 37, 2006

Birth-Death Models in Macroevolution

Sean Nee 1

The Posterior and the Prior in Bayesian Phylogenetics

Michael E. Alfaro and Mark T. Holder

Unifying and Testing Models of Sexual Selection

Hanna Kokko, Michael D. Fennions, and Robert Brooks

Genetic Polymorphism in Heterogeneous Environments: The Age of Genomics

Philip W. Hedrick

Ecological Effects of Invasive Arthropod Generalist Predators

William E. Snyder and Edward W. Evans. ...

The Evolution of Genetic Architecture

Thomas F. Hansen

The Major Histocompatibility Complex, Sexual Selection, and Mate Choice Manfred Milinski....

Some Evolutionary Consequences of Being a Tree Rémy 7. Petit and Arndt Hampe

Late Quaternary Extinctions: State of the Debate

Paul L. Koch and Anthony D. Barnosky

Innate Immunity, Environmental Drivers, and Disease Ecology of Marine and Freshwater Invertebrates Laura D. Mydlarz, Laura E. Fones, and C. Drew Harvell....

Experimental Methods for Measuring Gene Interactions Feffery P. Demuth and Michael F. Wade

Corridors for Conservation: Integrating Pattern and Process

Cheryl-Lesley B. Chetkiewicz, Colleen Cassady St. Clair, and Mark S. Boyce 
The Population Biology of Large Brown Seaweeds: Ecological

Consequences of Multiphase Life Histories in Dynamic Coastal

Environments

David R. Schiel and Michael S. Foster

Living on the Edge of Two Changing Worlds: Forecasting the

Responses of Rocky Intertidal Ecosystems to Climate Change

Brian Helmuth, Nova Mieszkowska, Pippa Moore, and Stephen 7. Hawkins

Has Vicariance or Dispersal Been the Predominant Biogeographic

Force in Madagascar? Only Time Will Tell

Anne D. Yoder and Michael D. Nowak

Limits to the Adaptive Potential of Small Populations

Yvonne Willi, Fosh Van Buskirk, and Ary A. Hoffmann

Resource Exchange in the Rhizosphere: Molecular Tools and the

Microbial Perspective

Zoe G. Cardon and Daniel 7. Gage

The Role of Hybridization in the Evolution of Reef Corals

Bette L. Willis, Madeleine 7.H. van Oppen, David 7. Miller, Steve V. Vollmer,

and David 7. Ayre

The New Bioinformatics: Integrating Ecological Data from the Gene to the Biosphere

Matthew B. Fones, Mark P. Schildhauer, O.F. Reichman, and Shawn Bowers

Incorporating Molecular Evolution into Phylogenetic Analysis, and a

New Compilation of Conserved Polymerase Chain Reaction

Primers for Animal Mitochondrial DNA

Chris Simon, Thomas R. Buckley, Francesco Frati, fames B. Stewart, and Andrew T. Beckenbach

The Developmental, Physiological, Neural, and Genetical Causes and

Consequences of Frequency-Dependent Selection in the Wild

Barry Sinervo and Ryan Calsbeek

Carbon-Nitrogen Interactions in Terrestrial Ecosystems in Response

to Rising Atmospheric Carbon Dioxide

Peter B. Reich, Bruce A. Hungate, and Yiqi Luo

Ecological and Evolutionary Responses to Recent Climate Change

Camille Parmesan

\section{Indexes}

Cumulative Index of Contributing Authors, Volumes 33-37 671

Cumulative Index of Chapter Titles, Volumes 33-37 